\title{
Rare Complication of Necrotizing Pancreatitis: Extension of Retroperitoneal Abscess into Femoral Region
}

\author{
Maja Karin ${ }^{1}$, Ante Bogut ${ }^{1}$, Ivan Romic ${ }^{2, *}$, Hrvoje Silovski², Josip Figl², Danijel Pravdic ${ }^{1}$, Mile Volaric$^{1}$, \\ Emil Babic ${ }^{1}$, Branko Bakula³, Renata Romic ${ }^{4}$
}

\begin{abstract}
Distant abscesses are uncommon during the episode of acute pancreatitis (AP). However, these are possible sequalae of necrotizing pancreatitis and should be treated appropriately to prevent serious septic complications. We demonstrate a case of a 56-year-old male patient who presented with severe necrotizing pancreatitis and distant retroperitoneal abscess that reached femoral region and was detected on diagnostic imaging scans. Combination of surgical and supportive therapy was employed, and the patient recovered well with no permanent consequences. Our article highlights the importance of quick and accurate diagnosis and timely intervention in this rare type of pancreatitis complication.
\end{abstract}

\section{KEYWORDS}

pancreatitis; retroperitoneal; drainage

\section{AUTHOR AFFILIATIONS}

${ }^{1}$ Internal Medicine Clinic with Dialysis Center, University Clinical Hospital Mostar, Bosnia and Herzegovina

2 Surgery Department, University Clinical Hospital Zagreb, Croatia

${ }^{3}$ Surgery Department, University Clinical Hospital Sveti Duh, Zagreb, Croatia

${ }^{4}$ Family Medicine Department, Health centre Zagreb, Zagreb, Croatia

* Corresponding author: Department of Surgery, University Hospital Centre Zagreb, Kispaticeva 12, 10000 Zagreb; e-mail: i.romic@gmail.com

Received: 7 June 2020

Accepted: 24 November 2020

Published online: 14 April 2021

Acta Medica (Hradec Králové) 2021; 64(1): 46-49

https://doi.org/10.14712/18059694.2021.8

(c) 2021 The Authors. This is an open-access article distributed under the terms of the Creative Commons Attribution License (http://creativecommons.org/licenses/by/4.0), which permits unrestricted use, distribution, and reproduction in any medium, provided the original author and source are credited. 


\section{INTRODUCTION}

Acute necrotizing pancreatitis (ANP) is a severe form of acute pancreatitis, characterized by necrosis of the pancreas and surrounding tissues with development of pancreatic collections and abscesses. It is associated with high rates of morbidity and mortality and long-term complications. Wide clinical picture, atypical presentation, local and systemic complications make treatment of acute necrotizing pancreatitis very challenging for both surgeons and gastroenterologists (1). Most severe complications of acute necrotizing pancreatitis are result of peripancreatic collections rich in digestive enzymes. It leads to necrosis of peripancreatic tissue and spreading of inflammation process along the retroperitoneum. The descent of necrotic retroperitoneal collections along the retroperitoneal space to the femoral region is a rare but possible manifestation of ANP that all clinicians should have on mind when evaluating femoral and inguinal swelling.

\section{CASE REPORT}

A 56-year-old male patient was admitted to the gastroenterology department because he had abdominal pain for the last 4 days, ascites and microcytic anemia. The patient had a history of heavy alcohol consumption. On physical examination, diffuse abdominal tenderness, ascites and ventral hernia were noted. C-reactive protein (CRP) was elevated to $88.9 \mathrm{mg} / \mathrm{L}$ (range 0-5), alkaline phosphatase $213 \mathrm{U} / \mathrm{L}$, gamma glutamyl transferase (GGT) to $93 \mathrm{U} / \mathrm{L}$ (range 9-35) and serum amylase to $345 \mathrm{U} / \mathrm{L}$ (range 28-100). Abdominal ultrasound showed ascites and inhomogeneous calcification of pancreas suspicious of acute pancreatitis. Upper endoscopy showed esophageal and gastric varices. Conservative therapy was initiated and included broad spectrum antibiotics (Meropenem $3 \times 1 \mathrm{~g}$ daily + Metronidazole $3 \times 500 \mathrm{mg}$ ), parenteral nutrition, analgesics, intravenous fluids and electrolyte corrections. However, seventh day of the hospitalization, laboratory

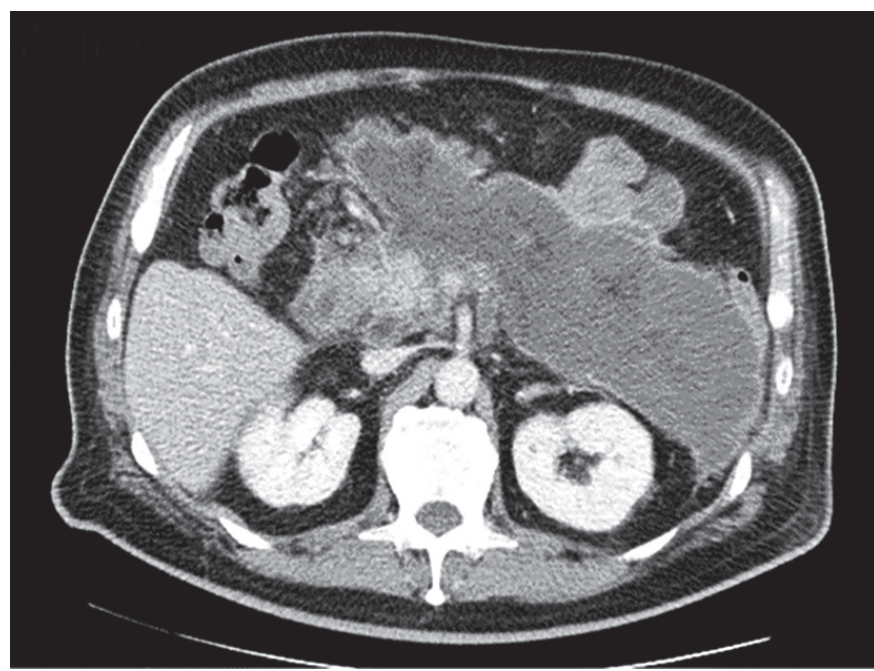

Fig. 1 MSCT showing a large retroperitoneal collection with abscess that occupied omental bursa, left pararenal and left iliacal retroperitoneal space with ascites intraperitoneally.

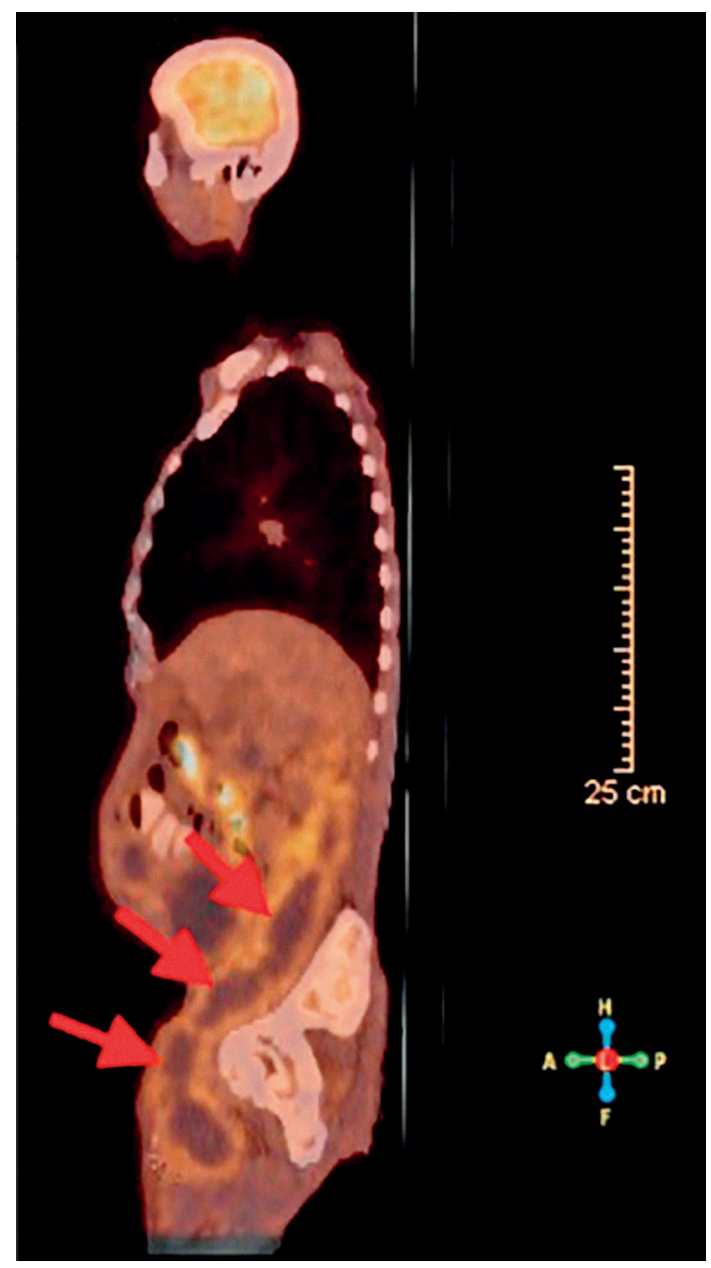

Fig. 2 PET/CT showing inflammatory process that represents communication between the retroperitoneal and left femoral region.

tests showed dramatic elevation of inflammatory markers: leukocytosis $\left(25 \times 10^{9} / \mathrm{L}\right)$ and CRP of $290 \mathrm{mg} / \mathrm{L}$ along with increase in serum and urine amylase, $3299 \mathrm{U} / \mathrm{L}$ and $4340 \mathrm{U} / \mathrm{L}$, respectively. The patient became febrile $\left(40^{\circ} \mathrm{C}\right)$ and developed large, painful bulge over the proximal part of right inguinal area and thigh.

Multi-slice computed tomography (MSCT) showed a large retroperitoneal collection with abscess that occupied omental bursa, left pararenal and left iliac retroperitoneal space with ascites intraperitoneally (Figure 1). Due to a suspicion of systemic septic foci, a positron emission tomography (PET/CT) was indicated and it showed continuous abscess formation between upper retroperitoneal space, femoral triangle and proximal part of the right thigh with no accumulation of F-fluorodeoxyglucose (FDG) in other parts of the body (Figure 2).

Abdominal paracentesis and right femoral region aspiration performed simultaneously showed $8374 \mathrm{U} / \mathrm{L}$ amylase in the ascites and 36,450 U/L amylase in the thigh collection. Microbiological analysis confirmed polymicrobial infection (E. Coli, Enterococcus spp., Klebsiella pneumoniae and Bacteroides spp.). All bacterial species were sensitive to initial antibiotic combination, so it was continued for the next 14 days.

Consultation with surgeon was done and it was decided to perform laparotomy, necrosectomy and surgical 
drainage of femoral collections because we have proven extensive infected pancreatic necrosis, femoral abscess and progression of septic complications. Minimally invasive approaches were not performed since interventional radiologist considered that it was not possible to adequately drain all collections percutaneously while laparoscopic techniques in our institutions were not at such high level to allow safe and sufficient laparoscopic pancreatic necrosectomy.

The patient was further treated with total parenteral nutrition, octreotide injections, transfusions of red blood cell concentrate, antibiotics and additional percutaneous intraabdominal abscess drainage on 6th postoperative day (POD). Patient's clinical condition improved, octreotide and parenteral nutrition were discontinued on POD 8 , he started oral feeding on POD 9 and he was discharged on 24th POD with completely healed femoral and abdominal incisions. At the 1-year follow-up, the patient was asymptomatic and there were no repercussions on pancreatic endocrine or exocrine function.

\section{DISCUSSION}

Based on the revised Atlanta classification, there is a clinical spectrum of AP presentations which can be categorized as mild, moderately severe, or severe (2). This division depends on presence of organ failure and systemic complications. Acute pancreatitis is most (in $80 \%$ of cases) often a mild disease and treatment consists of supportive care with fluid resuscitation, pain control and nothing by mouth, while the role of antibiotics therapy and parenteral nutrition in mild cases is still widely debated $(3,4)$. On the other side, less common, but more severe form of AP when more than $30 \%$ of the gland is affected by necrosis, is called necrotizing pancreatitis and it may lead to septic complications, multi-organ failure and death. Necrotizing pancreatitis may result in acute or subacute development of sterile or infected pancreatic necroses, abscesses or pancreatic fistulas while late complications include pancreatic pseudocyst and pancreatic failure (5).

American Gastroenterological Association (AGA) and American College of Gastroenterology (ACG) guidelines suggest that AP can usually be diagnosed based on clinical symptoms and biochemical evidence of pancreatitis while imaging tests should be performed only if there is clinical deterioration or diagnostic dilemma $(6,7)$. Treatment depends on the severity of the disease and different scoring systems have been used to assess it and predict outcome (e.g., Ranson criteria, Glasgow score, Imrie score), but still, the course of AP is unpredictable in some cases so the adequate monitoring of clinical and laboratory signs are mandatory. When systemic inflammatory response syndrome and/or organ failure is detected, the patient should be admitted to an intensive care unit.

Regarding recent WSES (World society of emergency surgery) guidelines, the surgery is indicated in abdominal compartment syndrome, acute ongoing bleeding when an endovascular approach is unsuccessful, bowel ischemia or acute necrotizing cholecystitis during acute pancreatitis, bowel fistula extending into a peripancreatic collection and as a continuum in a step-up approach after percutaneous/endoscopic procedure (8).

Previous guidelines included some other surgery indications which changed over time mainly due to development of minimally invasive methods. Thus, the presence of infected pancreatic necrosis should be managed by Percutaneous drainage as the first-line treatment (step-up approach), alternatively, transgastric endoscopic necrosectomy and video-assisted retroperitoneal debridement may be considered. Surgery is therefore reserved when such methods are not available or these do not produce the desired result $(9,10)$.

Other indications that are less well defined, but many surgeons still consider these when deciding on surgery timing are: persistence of pancreatitis in spite of maximal medical therapy, deterioration of one or more organ systems or sterile pancreatic necrosis involving $50 \%$ or more of pancreatic tissue (11).

Abscesses are usually located retroperitoneally around the pancreas, but may spread along retroperitoneal planes into abdominal wall, and less commonly into intraperitoneal space after erosion of peripancreatic structures. Because of such pathogenesis of pancreatic necrotizing processes, secretions may extend to unusual anatomical locations, presenting with clinical features which may mimic various other disorders $(12,13)$.

There are reports of pancreatic abscesses reaching psoas muscle (14), hepato-gastric ligament (15) or scrotum (16) but this is the first report of an abscess as consequence of pancreatitis that was located in femoral region and upper thigh.

In addition, we found reports on extra-pancreatic location of post-pancreatitis pseudocyst complications location (mediastinum, pleura, spleen, stomach wall), but these were late ( $>4$ weeks) complications of AP with proved pseudocyst morphology so pathogenesis and disease course of clinical scenario reported in our study was different and it occurred in early phase of $\operatorname{AP}(17,18)$.

We included octreotide in conservative therapy as some authors have suggested an indication for somatostatin and octreotide in the treatment of pancreatic fistulae. This is explained by its direct anti-inflammatory and cytoprotective effects as well as potent inhibition of exocrine secretion of the pancreas, which has an important role in the pathogenesis of acute pancreatitis (19).

Despite the rarity of distant pancreatic abscesses, gastroenterologists and surgeons should be aware of this clinical entity during the management of AP especially when unexplained septic complications or unusual abdominal, inguinal or femoral swellings develop.

\section{CONCLUSION}

The presented case highlights the need to consider important clinical challenges in diagnosis and therapy of acute necrotizing pancreatitis and its complications. 


\section{REFERENCES}

1. Bugiantella W, Rondelli F, Boni M, et al. Necrotizing pancreatitis: A review of the interventions. Int J Surg 2016; 28(Suppl 1): S163-71.

2. Banks PA, Bollen TL, Dervenis C, et al, for the Acute Pancreatitis Classification Working Group. Classification of acute pancreatitis - 2012 revision of the Atlanta classification and definitions by international consensus. Gut 2013; 62(1): 102-11.

3. Krishnan K. Nutritional management of acute pancreatitis. Curr Opin Gastroenterol 2017; 33(2): 102-6.

4. Isenmann R, Runzi M, Kron M, et al., for the German Antibiotics in Severe Acute Pancreatitis Study Group. Prophylactic antibiotic treatment in patients with predicted severe acute pancreatitis: a placebo-controlled, double-blind trial. Gastroenterology 2004; 126(4): 997-1004.

5. Kokosis G, Perez A, Pappas TN. Surgical management of necrotizing pancreatitis: an overview. World J Gastroenterol 2014; 20(43): 16106-12.

6. Baron TH, DiMaio CJ, Wang AY, Morgan KA. American Gastroenterological Association Clinical Practice Update: Management of Pancreatic Necrosis. Gastroenterology 2020; 158(1): 67-75.

7. Tenner S, Baillie J, DeWitt J, Vege SS, and the American College of Gastroenterology. American College of Gastroenterology guideline: management of acute pancreatitis. Am J Gastroenterol 2013; 108(9): $1400-15 ; 1416$.

8. Leppaniemi A, Tolonen M, Tarasconi A, et al. 2019 WSES guidelines for the management of severe acute pancreatitis. World J Emerg Surg 2019; 14(27): 1-20.

9. Greenberg JA, Hsu J, Bawazeer M, et al. Clinical practice guideline: management of acute pancreatitis. Can J Surg 2016; 59(2): 128-40.
10. van Santvoort HC, Besselink MG, Bakker OJ, et al., for the Dutch Pancreatitis Study Group. A step-up approach or open necrosectomy for necrotizing pancreatitis. N Engl J Med 2010; 362(16): 1491-502.

11. Working Group IAP/APA Acute Pancreatitis Guidelines. IAP/APA evidence-based guidelines for the management of acute pancreatitis. Pancreatology 2013; 13(4 Suppl 2): e1-15.

12. Vikram R, Balachandran A, Bhosale PR, Tamm EP, Marcal LP, Charnsangavej C. Pancreas: peritoneal reflections, ligamentous connections, and pathways of disease spread. Radiographics 2009; 29(2): e34.

13. Yu ES, Lange JJ, Broor A, Kutty K. Acute Pancreatitis Masquerading as Inferior Wall Myocardial Infarction: A Review. Case Rep Gastroenterol 2019; 13(2): 321-335.

14. Deshmukh S, Roberts K, Morris-Stiff G, Smith A. Pancreatico-psoas fistula: a rare complication of acute pancreatitis. BMJ Case Rep 2012; 2012: bcr1120115083.

15. Bakshi S. Pancreatic abscess within hepato-gastric ligament: case report of an extremely rare disease. BMC Surg 2020; 20(1): 20.

16. Ballestero DR, Aguilera TC, Gutierrez BJL, et al. Scrotal mass as result of a pancreatic pseudocyst extension. Actas Urol Esp 2008; 32: 261-4.

17. Mofredj A, Cadranel JF, Dautreaux M, et al. Pancreatic pseudocyst located in the liver: a case report and literature review. J Clin Gastroenterol 2000; 30: 81-3.9.

18. Aghdassi A, Mayerle J, Kraft M, Sielenkämper AW, Heidecke CD, Lerch MM. Diagnosis and treatment of pancreatic pseudocysts in chronic pancreatitis. Pancreas 2008; 36(2): 105-12.

19. Cavallini G, Frulloni L. Somatostatin and octreotide in acute pancreatitis: the never-ending story. Dig Liver Dis 2001; 33: 192-201. 